\title{
PREVENCIÓN DE LAS DISPUTAS INTERNACIONALES DE INVERSIÓN
}

\section{PREVENCÃO DAS DISPUTAS INTERNACIONAIS DE INVERSÃO}

Luis A. González García*

Resumen: El costo de los arbitrajes de inversión para la mayoría de los países en Latinoamérica es alarmantemente alto. En el aspecto financiero, los costos de procedimiento ascienden los millones de dólares y el riesgo de tener que pagar cientos de millones o incluso billones de dólares en materia de daños compensatorios. En el aspecto regulatorio el arbitraje de inversión no ha funcionado como instrumento de corrección en la toma de decisiones gubernamentales. Por estas razones, es tiempo para los países de la región de re-direccionar su política de atracción y protección a la inversión extranjera hacia la prevención de disputas internacionales en materia de inversión. El presente artículo propone una serie de mecanismos de colaboración y transparencia en las instituciones públicas. Estos mecanismos de prevención no deben ser sustitutos del arbitraje de inversión. El arbitraje inversionista-Estado debe verse como una herramienta más -y no la solución-de los gobiernos latinoamericanos en el fortalecimiento del estado de derecho y la seguridad jurídica en las relaciones económicas de los países.

Resumo: Os custos das arbitragens de investimento são, para a maioria dos países da América Latina, alarmantemente altos. Nos aspectos financeiros, os custos de procedimento superam milhões de dólares e há o risco de se ter que pagar centenas de milhões - ou inclusive bilhões de dólares por danos compensatórios. No aspecto regulador, a arbitragem de investimentos não funciona como instrumento de correção na tomada de decisões governamentais. Por estas razões, o tempo para que os países da região possam redirecionar sua política de atração e proteção ao investimento estrangeiro vai até a prevenção de disputas internacionais na matéria de investimentos. O presente artigo propõe uma série de mecanismos de colaboração e transparência nas instituições públicas. Estes mecanismos de prevenção não devem ser substitutos da arbitragem de investimento. A arbitragem investidor-Estado deve ser analisada como uma ferramenta mais - e não como solução-para os governos da América

* Matrix Chambers, Londres. E-mail: LuisGonzalezGarcia@matrixlaw.co.uk 
Latina no fortalecimento do Estado de Direito e da segurança jurídica nas relações econômicas dos países.

Palabras clave: Atracción a la inversión, Prevención, Alerta temprana Palavras-chave: Atração de investimento, Prevenção, Alerta precoce

\section{EL PROBLEMA CON LA SUSCRIPCIÓN DE TRATADOS DE INVERSIÓN}

El arbitraje de inversión contenido en los tratados de promoción y protección a la inversión o tratados bilaterales de inversión (“TBI") permite que los nacionales de un país exportador de capital cuenten con acceso directo al arbitraje internacional solo si el Estado incumple con estándares mínimos internacionales de protección a las inversiones extranjeras conforme al derecho internacional. El Estado receptor, por su parte, ávido de capital extranjero y no necesariamente con las mejores credenciales o historial ante la comunidad internacional en materia de estabilidad económica, política y jurídica (normalmente un país en vías de desarrollo) le conviene y acepta el pacto de someterse a la jurisdicción internacional toda vez que es la manera más rápida y menos costosa para un país (especialmente en vías de desarrollo) de mejorar su reputación hacia el exterior como un país "amigable" a la inversión extranjera. Llevar a cabo una reforma judicial, de desregulación económica, competitividad, reforma y fortalecimiento de las instituciones públicas con mayor transparencia en su actuar no sólo tiene un desgasto político interno importante, sino requiere tiempo y asignación de recursos considerables. El TBI parece ser la alternativa ideal de generar la reputación deseada que atraiga capital extranjero.

El suscribir o no un TBI, no es más que un proceso de costobeneficio que realiza todo Estado. Los Estados que suscriben tratados de inversión no le temen restringir su soberanía y someter sus actos al escrutinio de un tribunal internacional. De hecho, en algunos casos, lo fomentan. Esto es porque el Estado considera a un TBI como instrumento de política pública no sólo en cuestión de reputación sino que ayudará a inducir estabilidad en las relaciones económicas y despolitizar las decisiones en los proyectos de inversión. Es decir, es un compromiso del Estado a conducirse frente a inversionistas extranjeros de manera no discriminatoria y conforme al estado de derecho. En caso de no hacerlo, sigue la lógica, el Estado se obliga a pagar una indemnización por los daños económicos ocasionados por la conducta arbitraria. Este compromiso del Estado garantiza que no importa qué fuerza política esté en poder las reglas de juego ofrecidas al inversionista 
no será cambiadas de manera arbitraria. Ello tendrá como consecuencia natural el mejoramiento de la toma de decisiones del gobierno. Si no lo logra a través de la mera suscripción del TBI, lo hará a través del laudo arbitral. Así, aún si la mera existencia de una obligación internacional no es respetada en su momento por el Estado, por ejemplo, la obligación de no discriminar al extranjero, habrá un tribunal internacional que ayude a poner la casa en orden porque mediante la emisión de un laudo arbitral pondrá en evidencia el comportamiento irracional de un órgano o institución del Estado. A contrario de lo que se pudiera pensar, exhibir la conducta arbitraria de una autoridad no es necesariamente un punto negativo para el Estado. Por el contrario, el Ejecutivo u otros órganos lo utilizarán como una herramienta para que sus instituciones y demás instituciones públicas aprendan del error y corrijan su actuar para el futuro. En el interior de las estructuras de gobierno, un laudo será un instrumento de corrección, prevención y aprendizaje para las instituciones de qué hacer y no hacer en la toma de decisiones futuras en situaciones similares. Así, el esquema de protección a la inversión establecido en los TBI es uno donde -por lo menos en teoría- el beneficio es mayor que el costo. El inversor se protege, se despolitiza la disputa de inversión, se fortalecen los lazos de cooperación entre Estados, aumentan las oportunidades de negocio entre los países, mejora la reputación del país en vías de desarrollo, se fortalece el estado de derecho, se mejora la gobernabilidad del país dando por resultado, al final del eslabón, el desarrollo económico esperado del país.

De esta manera, al suscribir un TBI puede llegarse a pensar que el Estado envía un mensaje alentador de seguridad jurídica al capital extranjero en un mundo donde la competencia por la atracción a la inversión entre los países en desarrollo es cada vez más intensa. El TBI debía - para algunos - funcionar como un instrumento de atracción a la inversión. Por lo menos, eso se pensaba en los 1990s.

El problema es que el sistema no ha producidolos efectos esperados. El costo ha sido mayor que el beneficio. El hecho es que la suscripción de los tratados de inversión no ha sido la herramienta importante que algunos países esperaban. Cada vez hay más voces que confirman que no hay una correlación entre el aumento de la inversión extranjera directa y la suscripción de los tratados. Es más factible que la reputación y atracción de la inversión extranjera provenga de muchas otras fuentes como, por ejemplo, la membrecía a la OMC; los vínculos con bloques comerciales; el acceso a mercados internacionales, la apertura de ciertos sectores a la inversión privada, el clima laboral, infraestructura, los recursos naturales, capacidad de consumo interno y la apertura a la inversión extranjera de sectores estratégicos, entre otros. La conclusión es que no hay evidencia que los tratados hayan propiciado un aumento de los flujos de inversión en los países en desarrollo. 
Lo que ha venido en aumento a consecuencia de los tratados es el número de disputas internacionales en materia de inversión. Los arbitrajes de inversión cada vez son más complejos, largos y costosos ${ }^{1}$. Sólo en costos de procedimiento, un arbitraje le puede costar al Estado millones de dólares. En materia de daños compensatorios, los países del Mercosur están bajo el riesgo potencial de tener que pagar cientos de millones o incluso billones de dólares. Esto es causa de alarma en la región ya que la mayoría de países de Latinoamérica enfrentan no uno sino múltiples arbitrajes de inversión a la vez. En muchos casos los montos de daños reclamados equivale al presupuesto anual del Estado en materia de seguridad, educación o de salud. Para cualquier país incluso los desarrollados, los costos de defender un caso en un arbitraje de inversión son excesivos. La situación se agrava si observamos que la mayoría de los países demandados son economías relativamente pequeñas. Esto es preocupante.

Los arbitrajes internacionales no sólo absorben recursos económicos sustanciales sino que tampoco han logrado ayudar a corregir la toma de decisiones de las autoridades. Peor aún, el arbitraje en particular el laudo desfavorable para el Estado- no ha coadyuvado al fortalecimiento del estado de derecho ni han mejorado la comunicación o cooperación entre los diversos órganos y entidades de los gobiernos. Los mismos errores en la toma de decisiones gubernamentales tienden a repetirse una y otra vez aún en los casos en los que ha sido involucrado el mismo ministerio y con el mismo tipo de proyectos de inversión. Este fue el caso de México en los casos que involucró la autoridad federal del medio ambiente. Un análisis de los casos muestra que a pesar de contar con decisiones que critican y sancionan el actuar de las autoridades, los Estados continúan actuando de la misma manera frente a los inversores: incapacidad en la manera de enfrentar los problemas socio-políticos de la comunidad o de sectores sociales vulnerables; y una incongruencia del Estado en el ejercicio de sus facultades discrecionales de regulación. Por otro lado, los Estados que enfrentan reclamaciones internacionales continúan sufriendo de una escasa comunicación y cooperación entre las instituciones y órganos de los diferentes niveles de gobierno en la prevención y solución de los conflictos y problemas con inversores.

La pregunta fundamental que los países de Latinoamérica tienen que plantearse es cómo lograr los objetivos trazados de atracción y protección a la inversión, se minimice el riesgo potencial del pago de cientos de millones de dólares en compensación y que ayude a fortalecer

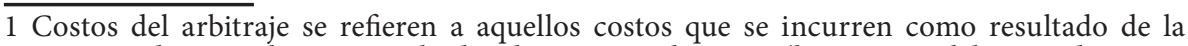
existencia de una disputa, incluidos los costos directos (los gastos del procedimiento arbitral, incluidos los gastos y honorarios de la secretaría y asistentes del tribunal, servicios de interpretación y traducción, honorarios y gastos de los árbitros, honorarios y gastos de la consultoría externa, pago de honorarios y gastos de expertos, consultores y peritos) y costos indirectos como salarios y gastos de viaje de los funcionarios públicos asignados a la disputa. 
el estado de derecho? La solución al problema es mantener un sistema costo-beneficio de atracción y protección a la inversión. El arbitraje no debe verse como la solución última al problema de protección a la inversión. Lo que se requiere es un cambio de dirección hacia la prevención de las disputas internacionales.

\section{LA IMPORTANCIA DE LA PREVENCIÓN}

\section{El costo-beneficio}

Un mecanismo de prevención de disputas es una actividad económica y política de gran importancia. Primero porque minimiza el riesgo de arbitrajes y segundo fortalece la toma de decisiones gubernamentales.

El arbitraje tiene repercusión de índole económica, jurídica y político para el Estado. Por un lado, el arbitraje absorbe recursos económicos importantes del Estado; no es mecanismo de solución de controversias accesible a todo inversor; y corre el riesgo de erosionar las relaciones entre las instituciones de gobierno e impedir la ejecución de las políticas de gobierno según un determinado plan de gobierno. Al evitar en la medida de lo posible recurrir al arbitraje se reducen los costos del Estado destinados a la defensa de los arbitrajes; se evita pagar sumas cuantiosas por pago de daños; se reducen los costos y riesgos políticos y sociales que conlleva mantener una disputa de inversión en sectores sensibles para el Estado como por ejemplo en materia de salud, medio ambiente, recursos naturales y servicios públicos. Segundo, porque un esquema de prevención crea y fortalecelos canales de comunicación entre las entidades de gobierno y facilita el entendimiento y cooperación entre las mismas. Al mantener un esquema de cooperación interinstitucional en donde diversas agencias formulan sus observaciones, tiene como resultado un mejoramiento en el ejercicio de toma de decisiones de las autoridades. Bajo un esquema de mayor transparencia, comunicación y cooperación gubernamental, el sistema de gobernabilidad mejorará y por ende se generará un clima favorable para la inversión.

En suma, los beneficios de la prevención de disputas son indiscutibles. La prevención pone énfasis en los procesos de cooperación, colaboración, participación pública y transparencia en las autoridades públicas. Entre más se fortalezcan estos procesos pre-litigiosos en el interior de los gobiernos, mejor serán atendidas las necesidades e intereses de las partes interesadas en conflictos relativo a un proyecto de inversión. Más aún, mantendrá las buenas relaciones con el inversor y abrirá futuras oportunidades de inversión. 


\section{LAS CAUSAS QUE ORIGINAN LAS DISPUTAS DE INVERSIÓN}

Para prevenir una disputa es necesario entender las causas que la generan. Obviamente, entender el origen de las disputas de inversión es una tarea complicada ya que cada proyecto de inversión tiene particularidades únicas que no se replican en otras áreas como por ejemplo las disputas en materia de construcción o transporte marítimo. Sin embargo, en casi todas las disputas internacionales de inversión podemos encontrar por la combinación de algunos de los siguientes escenarios.

El inversor y la autoridad mantienen una compleja relación en donde ambas partes persiguen intereses -en cierta medida- opuestos. Por un lado, el inversor tratará de maximizar sus ganancias al menor costo y en el menor tiempo. Para ello tratará en lo posible que la interferencia del Estado en su operación económica sea mínima. Por otro lado, el Estado estará interesado en salvaguardar el interés público por lo que estará propenso a interferir en la operación si es necesario. A mayor interferencia del Estado en la actividad económica del país, mayor la posibilidad de conflicto de intereses.

Por ejemplo, en proyectos de inversión en los sectores de infraestructura, turístico, extracción de recursos naturales, prestación de servicios públicos y medio ambiente, los proyectos involucran inversiones de larga duración, alto riesgo, inyección de capital importante y una operación fuertemente dependiente de una intensa regulación y supervisión del Estado. Los proyectos en estos sectores por lo general involucran tres etapas. La primera el diseño y licitación pública del proyecto. En esta etapa, la mayoría de los proyectos involucrará la participación de empresas extranjeras que quizá sea la primera vez que invierten en ese país y por lo tanto desconocen la cultura, prácticas y regulaciones de ese país. Por su parte, el Estado lanzará el proyecto de inversión normalmente como parte de un plan de gobierno y por lo tanto dicho proyecto deberá llevarse a cabo mientras dicho gobierno esté en poder de otra manera no podría garantizarse la continuidad del proyecto ni podrá ser presentado como un logro de ese gobierno. El tiempo no es un aliado del gobierno. El proyecto sigue su marcha sin que la comunidad en la que el proyecto se desarrolle haya sido consultada debidamente sobre el alcance, impacto y repercusiones del proyecto en la comunidad. Inicia un proceso de licitación. Los gobiernos por ley normalmente licitarán a aquel que ofrezca el precio más bajo. Ello significa que no necesariamente se le otorga a la empresa con mejores credenciales sino a la que ofrece el precio más barato. En este caso, los funcionarios públicos responsables del proyecto y de la licitación están consientes de que había mejores opciones en la mesa. Una licitación pública sea por invitación o abierta genera fuerte presión para la 
autoridad gubernamental. Aquella autoridad que licite será sujeta a posibles acusaciones de corrupción. Las acusaciones pueden venir de la oposición (fuerzas políticas) o la sociedad civil. Entre menos existan mecanismos de transparencia y auditoría hacia el interior del gobierno, las acusaciones sobre la comisión de irregularidades o corrupción aumentarán. En muchos casos será una cuestión de percepción de la opinión pública aún y cuando no haya fundamentos para ello.

Esto lleva a la segunda etapa del proyecto que es la negociación y suscripción de los contratos u otorgamiento de las licencias. Ya que el Estado ha licitado al precio más bajo y quizá convencido que puede ser sujeto a críticas por la sociedad civil, además de que quizá había mejores empresas que la que realmente ganó, la autoridad gubernamental que licita asegurará que los contratos contengan cláusulas de estricto y más alto cumplimiento por parte del operador. No son contratos que distribuyen el riesgo de manera equitativa. Así por ejemplo el riesgo de la operación correrá totalmente a cargo del inversionista y se incluirán cláusulas rigurosas de vigilancia, inspección, sanción y terminación del proyecto en caso de incumplimiento. En contraprestación, el operador negociará en el contrato una cláusula que le permite un margen de discreción para ajustar el precio del producto o servicio una vez en operación el proyecto de inversión. Esta etapa y por la magnitud de estos proyectos, se efectúa la firma de diversos protocolos, contratos y subcontratos no con una sino con diversas entidades del gobierno central y local a diferentes niveles de gobierno. Así, un solo proyecto puede involucrar la firma de compromisos por uno o varios ministerios, involucrando la firma de diversos funcionarios de diferentes agencias e instituciones y la creación de comités, subcomités y consultorías externas para la operación y supervisión del proyecto.

El éxito de la operación del proyecto de inversión dependerá en gran medida, no tanto de las reglas sino de los individuos que promovieron y negociaron el proyecto de inversión y las relaciones personales que se generen, por un lado, entre la autoridad y el inversor $y$, por el otro, entre las instituciones de gobierno. Un cambio de gobierno donde los individuos responsables de proyecto son reemplazados, se corre el riesgo de que la relación con el inversor se vea afectada. Hay una pérdida de confianza. El hecho de que un determinado proyecto de inversión sea impulsado como parte de un programa de un gobierno pudiera convertirse para el inversor de una bendición a su peor maleficio. Un cambio de gobierno de la oposición pudiera tener un efecto negativo en la viabilidad de la operación del proyecto de inversión.

La tercera etapa es la operación del proyecto de inversión. La manera en cómo un experimentado y sofisticado inversor extranjero opera con éxito en su país de origen no necesariamente se replicará en otro país con una cultura, idioma, nivel de bienestar social diferente 
y claridad en la regulación al de su país de origen. La manera de conducir una actividad empresarial no necesariamente funcionará de un país a otro. En muchos casos, los conflictos que afectan proyectos de inversión internacionales se focalizan en sectores poco regulados o muy regulados pero con poca supervisión o aplicación estricta de la ley en el país receptor de la inversión. Con escasa o nula regulación se dificulta el actuar del Estado porque la escasa regulación se traduce en compromisos o autorizaciones ambiguas o de difícil cumplimiento dadas la ambigüedad de la ley o las restricciones constitucionales o atribuciones de la administración pública central y local. Por otro lado, ello lleva a que la regulación y normativa evoluciona al mismo tiempo que la operación de la inversión. No es extraño observar cómo algunos proyectos inician operando de manera paralela con la adopción de nueva regulación tales como leyes, reglamentos, reglas de operación, manuales y lineamientos de operación. Esto inevitablemente aumenta el riesgo de conflictos entre el operador y la autoridad. Aunado a estos problemas, algunos sectores como el de medio ambiente quizá esté debidamente regulado o incluso regulado en exceso pero el problema radicará no en la falta de normativa sino en la escasa o nula aplicación y vigilancia en el cumplimiento de la misma. Ello permite que el inversionista relaje sus estándares de cumplimiento de las condiciones de la operación. Entre menos aplicación estricta a la ley el inversor llevará una tendencia a seguir relajando el cumplimiento de la normativa. Ello llevará a que un nuevo gobierno que observe este comportamiento y aún y cuando no sea severo el incumplimiento de la normativa aplicará sus facultades discrecionales para incluso dar por terminado el proyecto de inversión.

Por otro lado, el riesgo de un conflicto relacionado con una inversión aumentará si el proyecto de inversión afecta o involucra los derechos de sectores vulnerables como lo son comunidades indígenas o históricamente marginadas. El impacto de la explotación de recursos naturales en áreas donde se localizan las comunidades vulnerables ha tenido un impacto considerable en los arbitrajes de inversión. Estos han sido algunos de los factores y condiciones propensos a un conflicto de inversión. No importa el monto ni la magnitud del proyecto de inversión. Estos factores son comunes en la mayoría de los casos de inversión que involucran a países en desarrollo. Entre más sea el impacto del proyecto de inversión, mayor será el escrutinio de la sociedad civil y mayor el riesgo de una disputa. El riesgo aumenta cuando el inversor incurre en actos de corrupción durante la etapa de obtención de los permisos y concesiones o falsea información a la autoridad.

Solo si se entienden estos factores y elementos que causa un arbitraje de inversión se podrán tomar acciones preventivas que ataquen la raíz de los problemas de los proyectos de inversión. 


\section{LA PREVENCIÓN COMO RESPONSABILIDAD DEL SERVIDOR PÚBLICO}

No es interés ni del Estado ni del inversor recurrir al litigo internacional en disputas o conflictos que enfrenten en relación con un determinado proyecto de inversión. Por el contrario, es en el interés de ambas partes lograr una solución legal satisfactoria que convenga a todas las partes interesadas. Los tratados de inversión contemplan incluso la solución amistosa a través de consultas y negociación. Sin embargo, el hecho que sea interés de ambas partes llegar a una solución amistosa no debe interpretarse como un mero anhelo de la función pública. Cualquier orden constitucional y del funcionamiento de la administración pública de cualquier país tiene incorporado, sea implícita o explícitamente, el principio básico de cooperación. Este principio tiene su base en la entendido que cada gobierno es más eficiente, eficaz y consiente de las necesidades del gobernado cuando los funcionarios con capacidad de emitir actos de gobierno actúan en forma colaborativa. Una parte importante del trabajo colaborativo y coordinado de un gobierno es evitando futuros problemas o conflictos de manera constructiva. Este es un elemento inherente en el proceso de la toma de decisiones y de hacer política de un gobierno.

En ese sentido evitar el litigio es una obligación implícita del funcionario público. Las instituciones del Estado, en general, deben procurar en todo momento prevenir las disputas o conflictos con los gobernados. El objetivo de cualquier acto de autoridad es tomar en cuenta los intereses de todas las partes interesadas, valorar las implicaciones, reducir el riesgo del litigio y balancear los intereses en juego. Todas las instancias pre-litigiosas deben ser agotadas. Presentar alternativas de solución en cualquier situación de conflicto debe ser parte de la cultura de la autoridad. Desgraciadamente la prevención no es vista como una obligación positiva por muchos funcionarios públicos. Se percibe más como un anhelo, probablemente conveniente pero no asumido como una obligación positiva del servidor público. La percepción es equivocada. La prevención -incluida o no esta palabra mágica en el estatuto o reglamento interno de la estructura orgánica de la administración pública- debe el funcionario público asumirla como una obligación implícita en sus atribuciones. Esto es todavía más evidente para los ministerios que tienen dentro de sus facultades vigilar el cumplimiento de las obligaciones adquiridas por el Estado en materia de inversión. 


\section{5. ¿ES FACTIBLE UN ESQUEMA NACIONAL DE PREVENCIÓN DE DISPUTAS DE INVERSIÓN?}

Importa poco si el mecanismo de prevención tiene por objetivo evitar conflictos armados, ambientales, laborales o de inversión. Cualquier fórmula de prevención que involucre gobiernos enfrenta obstáculos institucionales y de operación. Esto es porque cada país tiene estructuras y mecanismos propios lo que hace que cualquier esquema nuevo tendría que ajustarse a la estructura ya existente. Lo que pudiera ser factible para un país pudiera no funcionar para otro. Para que funcione un modelo de prevención regional éste debe ser lo suficientemente flexible basado en ciertos principios básicos para que pueda ser incorporado en el trabajo diario de las estructuras, procesos e instituciones ya existentes hacia el interior de los gobiernos.

El éxito de una fórmula de prevención se logra mediante la identificación de actividades que vayan a la raíz del problema que origina una reclamación internacional de inversión. Es necesario igualmente reconocer las limitaciones prácticas que tienen los Estados para implementar mecanismos de acción preventiva. Es fundamental que ningún programa o sistema de prevención violente los límites y atribuciones constitucionales de cada entidad y órgano del Estado.

Otro elemento importante de cualquier sistema de prevención de inversión es que tiene que dirigirse a todo conflicto o problema relacionado con una inversión privada independiente de la nacionalidad del inversor. Así, ya sea un nacional o extranjero el esquema no debe discriminar. Cualquier inversor privado debe tener acceso a los mecanismos de prevención y cualquier autoridad gubernamental debe trabajar en la prevención por igual, sin distinción de nacionalidad. Esto es por razones prácticas y jurídicas. Primero, como una cuestión práctica, un esquema que sólo esté dirigido a la prevención de disputas para inversores extranjeros resulta ineficaz porque el objetivo debe ser mejorar los esquemas de coordinación y de toma de decisiones gubernamentales independientemente de la nacionalidad del actor económico involucrado. Segundo, resulta prácticamente imposible conocer la verdadera nacionalidad de un inversor. Pudiera ser que el inversor mantenga una inversión en el país con mayoría de acciones nacionales y sin embargo la empresa esté controlada directa o indirectamente por no nacionales. La autoridad por lo general desconocerá la verdadera nacionalidad y para el objetivo que se pretende lograr en la prevención que es mejorar la toma de decisiones gubernamentales, la distinción de nacionalidad resulta irrelevante. Lo importante es limpiar la casa desde adentro. Tercero, desde el punto de vista jurídico pudiera el Estado otorgando un trato discriminado a nivel local al extranjero sobre al nacional lo cual pudiera ser inconstitucional. 
Por último, un esquema de prevención en inversión requiere no sólo del esfuerzo del gobierno. El inversor debe asumir su parte. Para ello debe incentivarse un cambio en la conducta de los países en vías de desarrollo y más aún importante en aquellos países o regiones donde la inversión se localiza en comunidades o poblaciones vulnerables. El éxito de una inversión ya no puede medirse exclusivamente a través de medidores económicos. Las ganancias o utilidades no debe ser el único medidor del éxito empresarial. El mundo ha cambiado en los últimos 30 años. El desarrollo económico y las políticas de liberalización económica ya no son vistos de manera aislada con compromisos sociales tendientes a la promoción, respeto y desarrollo de los derechos humanos, medio ambiente y desarrollo social. En esta nueva etapa, todo inverso deberá empezar a asumir estándares de responsabilidad corporativa o inversión social en la comunidad en la que invierte.

Si se mantienen este enfoque para la aplicación de una estrategia de prevención de disputas de inversión será factible y realista adoptar un programa de prevención de disputas de inversión en los países de Latinoamérica.

\section{PRINCIPIOS BÁSICOS}

El objetivo de la prevención es evitar o minimizar el riesgo de arbitrajes internacionales de inversión. La mayoría de las disputas pueden ser evitadas si la acción preventiva es oportuna. Cualquier acción preventiva del Estado debe basarse en tres objetivos principales: i) prevenir el surgimiento de un conflicto en proyectos de inversión; ii) prevenir que un conflicto se transforme en una disputa de inversión; y iii) minimizar el riesgo de la responsabilidad internacional del Estado.

Una estrategia efectiva de prevención sólo se logrará si: i) se implementa un esquema que facilite la integración y coordinación entre las autoridades en la toma de decisiones; y ii) se genere una cultura de la prevención en el gobierno.

La mayoría de los arbitrajes de inversión involucran asuntos de alta sensibilidad política para el Estado por lo que se requiere de un manejo profesional, neutral, de asesoría especializada en materia de inversión y con habilidades especiales para el manejo de conflictos. Por ello, es importante que cualquier esquema de prevención a implementarse en un país de Latinoamérica cuente con la asesoría y apoyo externo. Un programa de acción preventiva eficaz funcionará si se lleva a cabo y de manera conjunta en el plano nacional con la asistencia internacional. 


\section{CONSTRUYENDO UN MECANISMO}

Si el Estado está convencido que la prevención de disputas de inversión es una actividad necesaria para mejorar el clima de inversión en el país, la siguiente interrogante es ¿cómo implementar un programa la prevención cuando no existen los mecanismos internos ni los recursos humanos y económicos en el país? ¿Cómo se puede desarrollar una cultura de la prevención en el seno del gobierno? ¿Cómo abrirlo a los gobiernos locales? ¿Dónde empezar?

Lo que propongo es una fórmula de acción preventiva que requiere el trabajo en dos frentes: nacional y regional. Para que una acción de prevención sea efectiva a nivel local se requiere de un enfoque práctico y global. No existe un mecanismo de prevención uni-talla. Cada Estado deberá implementar un mecanismo adecuado sus realidades y necesidades. Ahora bien, la realidad es que la mayoría de los países difícilmente pueden implementar un programa de prevención por sí solos. Las limitaciones de los Estados son obvias. La gran mayoría no cuentan con los recursos económicos y las capacidades humanas para tal empresa. Por ello es importante del apoyo concertado de organismos internacionales o regionales o académicas que asistan a los Estados. Un paso importante sería la de contar con un centro de asesoría legal especializado en materia de inversión en una región, por ejemplo, el Mercosur o Sudamérica enfocado en la prevención.

Si bien un mecanismo de prevención a nivel nacional podrá variar según las particularidades de cada país, la fórmula deberá basarse en ciertos pilares básicos. La fórmula requiere de: 1) análisis; 2) respuesta; y 3) cultura de la prevención. El análisis implica la capacidad de monitorear los proyectos o acciones que lleve o pretenda llevar a cabo alguna entidad gubernamental y emitir opiniones. En algunos casos, el análisis no será suficiente y requerirá de una respuesta oportuna a un problema específico. Aquí entra el segundo elemento: la "respuesta" que se traduce en la toma de acciones concretas para evitar que un problema existente se convierta en un conflicto. Es decir, la "respuesta" toma el lugar del "análisis" cuando ya el problema deja de ser un escenario probable de conflicto a uno donde es inminente el conflicto, si no se toman acciones oportunas. Las actividades de "respuesta" incluyen la facilitación, negociación y diálogo entre los actores involucrados. Ninguno de estas dos etapas tendrá éxito si no existe una cultura arraigada de la prevención. La cultura de la prevención requiere de un esfuerzo conjunto entre todos los niveles de gobierno. La cultura de la prevención es un trabajo permanente de educación e impulso político. Para ello se requiere de la implementación de un programa de capacitación permanente y asesoría a los funcionarios públicos a nivel municipal y central con el apoyo de instituciones internacionales o 
regionales.

\section{LA FÓRMULA PARA PREVENIR DISPUTAS}

Son varios pasos que se requieren tomar para implementar un mecanismo efectivo de prevención de disputas internacionales de inversión. Este artículo sugiere una serie de pasos que deben seguir los gobiernos y los inversores para evitar recurrir al arbitraje en sus disputas de inversión. La acción preventiva que se propone requiere en primer lugar implementar una estructura a nivel institucional. Una vez que se cuente con la infraestructura necesaria y adecuada a las particularidades de cada país, el siguiente paso es la implementación de una serie de estrategias en la operación de la prevención.

\subsection{Capacidad institucional}

Lo primero que se requiere es construir la capacidad institucional para la prevención de conflictos a través de la integración y cooperación intergubernamental. Establecer la capacidad institucional se requiere de voluntad política, toma de conciencia que las disputas en proyectos de inversión son inevitables, implementación de un sistema con una estructura permanente, desarrollo de programas y procedimientos de colaboración y coordinación e inversión en recursos humanos a través del entrenamiento y capacitación de funcionarios públicos. Los pasos a seguir son los siguientes:

\section{Voluntad política}

Un plan de prevención de disputas de inversión debe ser responsabilidad de los gobiernos centrales. Para ello se requiere de voluntad política. Ningún plan de prevención será eficaz si no hay un impulso político de alto nivel en el gobierno. Es importante que a más alto nivel se reconozca el reto que es para el Estado regular en el interés público y enfrentar el riesgo de una reclamación internacional de inversión con los costos que involucra el arbitraje internacional. Para enfrentar este reto de manera eficaz, es importante que exista un compromiso político de alto nivel, primero, entre los ministerios del gobierno central y posteriormente -en una segunda etapa- el gobierno central con los gobiernos locales para desarrollar estrategias que, en la medida de lo posible, minimicen el riesgo de conflictos y disputas con inversores y generen oportunidades de cooperación y colaboración en la solución de conflictos. Para ello se recomienda que a más alto nivel, preferentemente la Presidencia emita un comunicado, circular o decreto (según las condiciones de cada Estado) dirigido a las agencias 
e instituciones del gobierno central alentando el uso de mecanismos y procesos preventivos de conflictos en proyectos de inversión, particularmente en los siguientes sectores: agricultura, energía, turismo, salud, infraestructura, medio ambiente y servicios públicos concesionados.

\section{Designar una Agencia}

Para enfrentar el reto de prevención de disputas de manera eficaz se requiere que una agencia neutral e independiente sin facultades regulatorias dentro del gobierno central se designe como agencia líder del programa de prevención de disputas. La agencia será más eficaz si pertenece al ministerio encargado de promover la inversión extranjera y/o la implementación de los tratados de protección a la inversión. En algunos países le corresponderá al ministerio de comercio y en otros casos al de relaciones exteriores. Lo importante es que sea una agencia despolitizada en la medida de lo posible. Un elemento importante es el de la percepción. Es importante que el inversionista y la autoridad que regula el sector en el cual el inversor siente que ha sido afectado, vean a la agencia como una unidad neutral, de carácter técnico, profesional, de alta calidad moral, imparcial cuyo compromiso sea no la de favorecer a un determinado gobierno sino la de promover la inversión y a vigilar el cumplimiento de las compromisos internacionales asumidos por el Estado en los tratados internacionales.

La agencia deberá tener como función primaria asesorar a las instituciones, órganos y entidades públicas respecto a la compatibilidad de las políticas, prácticas y regulaciones internas dirigidos a los proyectos de inversión que pudieran ser violatorios de los compromisos internacionales del Estado en materia de inversión. La agencia deberá, en particular:

-monitorear la actividad legislativa del gobierno central incluyendo la revisión y análisis de iniciativas de ley, reglamentos o cualquier regulación o práctica que pudiera ser incompatible con las obligaciones internacionales del Estado en materia de protección a la inversión;

-evaluar los proyectos de inversión extranjera, incluidos las licitaciones públicas;

-proporcionar un foro horizontal para la resolución de conflictos y problemas en proyectos de inversión. La unidad deberá contar dentro de sus funciones facilitación del diálogo, negociación y mediación entre las partes en conflicto;

-brindar capacitación y entrenamiento a las demás instituciones y órganos del Estado sobre técnicas de prevención de disputas y contenido de las obligaciones internacionales del Estado en 
materia de inversión.

\section{Identificar las necesidades internas}

El ministerio al que dependa la agencia designada deberá convocar a una reunión de alto nivel con los ministerios del gobierno central encargados de regular los sectores de energía, agricultura, comunicaciones, turismo, medio ambiente, salud e infraestructura. Las reuniones que se lleven a cabo tendrán como objetivo discutir de manera preliminar las directrices y estrategias de un programa intergubernamental de coordinación y cooperación así como identificar las áreas de posible conflicto en proyectos de inversión extranjera.

En seguimiento a las reuniones, la agencia deberá llevar a cabo una encuesta en aquellas instituciones del gobierno central seleccionadas que tengan atribuciones específicas para regular la inversión en los sectores como el de agricultura, energía, salud, medio ambiente, infraestructura y turismo. El objetivo de las encuestas tiene un doble propósito: i) establecer formalmente un diálogo de coordinación interinstitucional $y$, ii) identificar debilidades y fortalezas de los esquemas de prevención, en caso que existan. Las respuestas a las encuestas deberán dar pauta para elaborar de manera específica esquemas de coordinación y cooperación.

\section{Implementar mecanismos de prevención}

Dependiendo de las propuestas que resulten de las reuniones interinstitucionales, modificaciones a la legislación deberán llevarse a cabo. El objetivo debe ser atacar el problema desde la raíz. Un análisis de los arbitrajes de inversión evidencia la necesidad de los Estados de contar con un esquema de cooperación, consulta y coordinación efectiva entre las entidades del gobierno a fin de llevar la acción de la autoridad sea una decisión debidamente informada sobre las implicaciones jurídicas, técnicas y políticas de llevar cabo la medida. Para ello los Estados requieren re-evaluar el marco legal de decisión política, aplicación de la ley y transparencia que englobe un esquema de colaboración gubernamental de toma de decisiones. La agencia de prevención deberá tomar un rol activo. Primero deberá establecer una hoja de ruta en la que promoverá la suscripción de acuerdos entre las distintas instituciones del gobierno central a fin de que se establezcan los mecanismos y métodos de alimentación de información y seguimiento a los problemas o conflictos que se susciten en los proyecto de inversión. Cada institución del gobierno central invitada a participar en el sistema de prevención designará un punto de contacto con la agencia. La agencia determinará la conveniencia y posibilidad de establecer mecanismos 
similares con las autoridades locales. Cualquier mecanismo que se implemente deberá ser permanente.

Segundo, la agencia deberá establecer un portal de internet que facilite y promueva la utilización de los mecanismos preventivos y en particular hacerlo accesible a los inversores para que ellos mismos puedan alertar a la agencia de cualquier asunto que pudiera convertirse en una disputa de inversión. A fin de evitar un abuso en la utilización del sistema de prevención, la agencia podrá restringir el acceso al sistema a proyectos de inversión en ciertos sectores y sujeto al cumplimiento de ciertos requisitos que acrediten que efectivamente se trata de un proyecto de inversión. Con el fin de dar mayor difusión y utilización del sistema deberán ser informadas las embajadas, cámaras de comercio, organismos empresariales y los bufetes jurídicos más representativos en el país.

Por último, se requiere contar con convenios entre la agencia y las instituciones con el objeto de fomentar la integración y comunicación entre las agencias mediante talleres, reuniones y mesas redondas de discusión que fortalezcan la colaboración interinstitucional.

\section{Asistencia internacional}

La gran mayoría de las disputas de inversión involucran temas de una gran sensibilidad política cuyas repercusiones pudieran afectar las relaciones políticas, económicas y sociales en el interior del Estado. En la mayoría de los casos las disputas de inversión involucran acciones del propio gobierno central y peor aún evidencian una ruptura o intereses encontrados entre las propias instituciones del gobierno. Una agencia de prevención que pertenece a una institución del gobierno podrá ser vista dentro de la estructura del gobierno como una entidad bajo órdenes del ministerio o gobierno al que pertenece por lo que sus opiniones y acciones para prevenir una disputa podrán verse como una interferencia inapropiada de un ministerio que persigue sus propios intereses. La credibilidad de una agencia de prevención podrá verse afectada por las acciones del ministerio mismo al que pertenece. En estos casos la asistencia de un órgano independiente es importante. La realidad de los gobiernos es que es más probable que una propuesta de acción preventiva sea más eficaz si la misma proviene de un organismo o persona independiente que del propio gobierno. La opinión que provenga de una entidad que goce de buena reputación, neutral, imparcial y ajena al gobierno tendrá más fuerza que aquella que provenga del interior del gobierno. Por ello recomiendo la creación de un centro de asesoría legal de inversión que asista a los gobiernos de Sudamérica en la prevención de disputas.

La creación de un centro de asesoría en materia de arbitraje de 
inversión ha tenido eco en diversos países latinoamericanos y esfuerzos importantes se han llevado a cabo para su puesta en marcha con la ayuda $\mathrm{y}$ asistencia de diversas organizaciones internacionales. La idea de que un centro incluya dentro de sus funciones servicios de representación legal en los arbitrajes genera problemas prácticos que dificultarán en el corto plazo la viabilidad de un centro de asesoría. Lo ambicioso del proyecto es quizá la razón por la que no ha podido ponerse en marcha. El centro que propongo no abarca la defensa de los Estados en el arbitraje. Tomando un enfoque práctico y realista, lo que se requiere y puede implementarse en un tiempo relativamente corto es un centro de asesoría que asuma como responsabilidad primaria la asistencia de los países en desarrollo en la prevención de disputas.

El centro de asesoría deberá ofrecer a los países de la región: 1) asesoría legal en materia del derecho internacional de las inversiones; 2) asistencia técnica en el diseño e implementación de mecanismos de prevención incluido un sistema de alerta temprana; 3) revisar y formular opiniones sobre casos, acciones o proyectos de regulación que afecten a proyectos de inversión y que le sean remitidos por las agencias nacionales de prevención; y 4) capacitación y entrenamiento en arbitraje de inversión y en el manejo de conflictos, incluido el desarrollo de técnicas de negociación, solución de conflictos y análisis de riesgo.

La creación y operación de un centro de asesoría requerirá la participación y asistencia de diversas organizaciones internacionales y posiblemente el respaldo de entidades académicas de prestigio.

\section{Recursos}

De poco sirve la voluntad política para impulsar un programa de prevención si el Estado no cuenta con los recursos necesarios para llevar a cabo la prevención en el aspecto operativo. Uno de los mayores desafíos que enfrentará cualquier país en desarrollo interesado en la prevención de disputas de inversión es la falta de recursos económicos.

Sin un presupuesto adecuado, habrá pocos incentivos para contratar a personal calificado, habrá restricciones de viaje, imposibilidad de organizar y financiar talleres y capacitación necesaria en el país, entre otras.

El Estado con asistencia internacional, probablemente a través del centro de asesoría, deberá invertir en la prevención. El financiamiento internacional es fundamental. A nivel doméstico la forma de obtención de recursos podría ser a través de la asignación de cierto porcentaje que el Estado destina a la implementación y administración de los tratados internacionales en materia económica.

Sólo si se invierte en recursos humanos y en financiamiento de seminarios y de capacitación y talleres será eficaz el programa de 
prevención.

\subsection{Prevención operacional}

\section{Alerta Temprana}

Los ministerios del gobierno central deberán establecer un mecanismo de alerta temprana de resolución de conflictos relacionados con un proyecto de inversión. La alerta temprana es un mecanismo basado en el principio de transparencia que tiene por objeto mejorar la capacidad de cada parte en un conflicto de conocer y tomar en cuenta los intereses de las otras partes interesadas en una etapa inicial en la toma de decisiones de política pública, legislativa y regulatoria, sin que para ello afecte o limite la autonomía de la autoridad en la toma de decisiones. La alerta temprana en la prevención de disputas de inversión deberá guiarse por los siguientes principios: 1) No discriminatorio. El sistema deberá estar disponible por igual a inversiones privadas nacionales o extranjeras; 2) Cooperación. Debe existir un mecanismo de cooperación institucional en dos niveles. Por un lado una red de cooperación de prevención entre los organismos internacionales y las nacionales, y por el otro, las redes de cooperación entre la agencia y los puntos de contacto de las instituciones públicas; y 3) Incluyente. Una acción preventiva temprana debe asegurar que todos los factores y posiciones han sido valorados a fin de que la autoridad pueda tomar una decisión razonada y debidamente informada.

Los objetivos de la alerta temprana son:

(i) Evaluar cualquier política o regulación que tenga un impacto en un proyecto de inversión. Ello incluirá cualquier medida o riesgo de acción política por parte de entidades locales o del gobierno central;

mantener una comunicación directa a nivel técnico y político entre el ministerio encargado de la regulación del sector y la agencia de prevención.

(ii) Cualquier problema o conflicto relativo a un proyecto de inversión independientemente del tamaño o nacionalidad del proyecto debe de someterse a la atención de la agencia.

(iii) La agencia deberá implementar procesos de seguimiento y supervisión de los problemas o conflictos alertados.

(iv) La agencia deberá servir como facilitador entre las partes en disputa; promover el diálogo; consultas y negociación; y, en su caso, ofrecer alternativas o propuestas de solución.

La alerta temprana requiere de las siguientes acciones:

(i) Identificar los sectores en donde exista mayor riesgo de enfrentar problemas con proyectos de inversión (puntos de conflicto). En 
países con economías pequeñas no deberá ser difícil encontrar los puntos en conflicto. Tradicionalmente será en las áreas donde se concentre la inversión privada en el país y la operación es de interés público ${ }^{2}$.

(ii) Identificar las condiciones propensas a conflictos. Los ministerios responsables de cada sector seleccionado deberán elaborar una lista de los proyectos de inversión que pudieran ser vulnerables a una disputa de inversión.

(iii) Involucrar al mayor número de actores en el sistema de alerta temprana. Las organizaciones no gubernamentales (ONGs), asociaciones agrícolas o laborales, embajadas, líderes políticos, empresarios, autoridades locales y la prensa cuentan con capacidad para alertar a la autoridad de manera oportuna. Normalmente será una ONG o sociedad civil la primera en percatarse y actuar cuando surge un conflicto involucrando un proyecto de inversión de alto impacto en la comunidad. Por ejemplo la mejor señal temprana de un conflicto en el sector del medio ambiente es cuando una ONG se moviliza. La agencia deberá mantener un canal de comunicación directo y fluido con las ONGs más importantes del país.

(iv) Escuchar a todos los actores involucrados. La agencia recomendará una acción preventiva temprana con base en la información que disponga. Es posible que la información que le llegue a la agencia sea imprecisa, incompleta o poco balanceada. Ello es porque cada parte en el conflicto relativo a un proyecto de inversión defenderá sus propios intereses. Así, la autoridad justificará su actuar y será selectivo en el suministro de documentos hacia la agencia. El inversor hará lo mismo, igual que las ONGs tendrán su propio interés en la disputa y por tanto su información podrá no ser del todo completa. Todo ello complicará la labor de la agencia en la propuesta de una solución preventiva. Por ello, será importante que el Estado cuente con legislación moderna de acceso a la información y transparencia así como la puesta en marcha de mecanismos de intercambio de información y coordinación de buena fe entre las instituciones del gobierno.

(v) Cooperación política. La solución a un conflicto relativo a un proyecto de inversión involucra no sólo el aspecto técnico de la disputa, sea cumplimiento de la legislación ambiental, criterios de entrega, calidad y pago, o incumplimiento de una cláusula

2 En el sector minero la extracción y explotación de recursos minerales, licencias y permisos que afecten el medio ambiente, extracción y distribución de petróleo y gas, concesión de servicios públicos primarios, proyectos de infraestructura, concesión de la operación en el área de telecomunicaciones, transporte aéreo, terrestre y marítimo; sector financiero. 
contractual. En la mayoría de los arbitrajes de inversión la materia objeto de la disputa rebasa el aspecto técnico. Sea cual sea la disputa de inversión, el alcance o desenlace de la disputa generalmente tendrá una dimensión política. Es por ello importante involucrar en la acción preventiva, según sea el caso, a la unidad del gobierno central encargada del diálogo y concertación política que asista a las partes en disputa en la solución del problema.

(vi) El rol como facilitador. Una función de la agencia será la de fungir como facilitador o ser quien designe al facilitador para un caso concreto si así lo disponen las partes en disputa. El objetivo del facilitador es asistir a las partes a fin de que logren un arreglo mutuamente satisfactorio. Sujeto a lo que dispongan las partes en disputa, el facilitador deberá identificar las cuestiones controvertidas y las no controvertidas, investigar los hechos, obtención de información y asistir a las partes en su diálogo.

\section{Capacitación}

La acción preventiva funcionará si existe un cambio de actitud de las instituciones de gobierno. Es imperante que exista una cultura de la prevención y ésta se logra mediante voluntad política y a través de un proceso continuo de capacitación. Para poder capacitar a las instituciones públicas es necesario que la propia agencia de prevención tenga las capacidades humanas y económicas para llevar a cabo tal función. La credibilidad y éxito del mecanismo de prevención mucho dependerá de la reputación con la que cuente la agencia dentro del gobierno. Es imprescindible la calidad en las actividades de prevención de la agencia. Ello requiere que quien forme parte de la agencia de prevención esté debidamente capacitado. Ello requiere de personal con ciertas habilidades y capacidades. Es importante que el individuo encargada de la agencia sea una persona con capacidad analítica y goce de buena reputación dentro gobierno.

Una vez que se cuente con la persona o el personal encargado de la agencia de prevención, deberá iniciarse un programa de entrenamiento y capacitación en prevención de disputas de inversión. La capacitación tendrá eficacia si va enfocado a una oficina o departamento específico. Un gobierno estará malgastando sus recursos si diseña un programa de prevención en donde el objetivo es exclusivamente impartir conferencias a audiencias abiertas al público en donde, por ejemplo, se explique el contenido de las cláusulas de expropiación y trato justo y equitativo a, por ejemplo, estudiantes, abogados y empresarios. Por el contrario, un curso de capacitación sobre prevención en un grupo selecto de funcionarios públicos, por ejemplo, del departamento de jurídico y la unidad que otorga licencias y permisos, el impacto será mayor. 


\section{El rol del inversor}

La inversión extranjera en países en desarrollo presenta oportunidades lucrativas de negocio pero también expone al inversor a riesgos, entre ellos, la privación del beneficio de su inversión por actos atribuibles al Estado. Dicha privación será la pólvora que encienda una disputa de inversión. Independientemente de si es justificada o no la interferencia del Estado en el proyecto de inversión, lo cierto es que en algunos casos la conducta del inversor pudo haber jugado un rol fundamental en la prevención de la disputa de inversión.

$\mathrm{Al}$ invertir en un país en desarrollo, los inversores normalmente siguen un mismo patrón de conducta. Primero, en aras de obtener un contrato lucrativo con un gobierno enfocan su atención en obtener el apoyo incondicional del gobierno en el proyecto y de obtener cierto tipo de "garantías" o "compromisos" que le garanticen que su inversión será una operación económica exitosa. Ello incluye actos o pagos indebidos a funcionarios, que el inversor considera clave para el éxito de su operación. En este proceso, ciertos inversores restarán importancia o simplemente ignorarán los riesgos inherentes al proyecto de inversión ${ }^{3}$.

Segundo, los inversores en sectores sensibles normalmente adoptan una postura de indiferencia hacia la comunidad que posiblemente será afectada por las actividades que desarrollará el inversor. El riesgo de conflictos es más alto cuando los inversores llevan a cabo sus operaciones en aéreas que afectan a sectores vulnerables de la sociedad. Así sea en la explotación de recursos naturales o la prestación de servicios públicos, los inversores, (especialmente las empresas multinacionales) han optado por no involucrar a la comunidad local en el proyecto; no mantenerla debidamente informada sobre las operaciones; o no contribuyen en el desarrollo social de dicha comunidad. Ya que el Estado difícilmente le exige al inversor mantener un compromiso social con la comunidad local, el inversor opta por mantenerse aislado de la comunidad. En última instancia, desde la perspectiva del inversor, la inversión sólo rinde cuentas a sus accionistas.

Ciertos arbitrajes evidencian el poco interés de los inversores en crear, apoyar, mantener y salvaguardar los intereses sociales de las comunidades en las que el proyecto de inversión opera. Este distanciamiento sin embargo sólo juega en contra del inversor. Los inversores deben entender el valor agregado que significa mantener a la comunidad como aliada. Me refiero a la comunidad y no a los líderes de esas comunidades. Si el inversor se comprometiera más con la comunidad en su conjunto el resultado sería un menor riesgo de que su inversión se vea afectada por presiones políticas o sociales. Sería más difícil para un grupo de presión atacar la reputación del inversor

3 Los riesgos asociados incluyen la inestabilidad política, económica y social del país. 
cuando éste mantiene un vínculo y alianza con dicha comunidad. La oposición hacia la inversión aún y cuando se hayan cometido algunas irregularidades en la operación sería reducida si el inversor coadyuva y está comprometido con el desarrollo social de la comunidad y la protección al medio ambiente.

Vivimos hoy en un mundo en donde la sociedad y la comunidad internacional demandan un cambio en la manera en cómo se conducen las empresas multinacionales ${ }^{4}$. Cada vez más observamos prácticas corporativas que reflejan valores y códigos de conducta asociados al desarrollo social, el respeto a los derechos humanos y el medio ambiente. Estos elementos están asociados con el concepto de responsabilidad social corporativa. Ello significa que el inversor tiene un rol en ayudar los objetivos de desarrollo social en la comunidad en la que operan. Los proyectos de inversión deben reflejar estos principios. Los gobiernos pueden regular las actividades de las empresas que operan en sectores vulnerables de la sociedad a fin de que los compromisos asumidos por el inversor reflejen el impacto ambiental y social en sus operaciones.

De igual manera las empresas deben conducirse de buena fe en los países en desarrollo y abstenerse de participar en prácticas inmorales o ilegales. Ello incluye la prohibición de soborno en el extranjero. Estados Unidos, Canadá y prácticamente todos los países de Europa en acorde con la Convención de la OECD han adoptado legislación que prohíbe y sanciona las prácticas de corrupción de sus empresas cometidas en el extranjero ${ }^{5}$.

La prevención juega un papel importante en cada uno de los ciclos de vida de la inversión extranjera. En la etapa pre-inversión, el inversor deberá tomar en cuenta lo siguiente:

(i) Tomar en cuenta los riesgos inherentes al proyecto de inversión en el país de que se trate, independientemente de cualquier promesa de ciertos funcionarios públicos obtención de grandes utilidades.

(ii) Revisar la legislación local y cumplir con los requerimientos municipales y estatales por igual.

(iii) En un proceso de licitación pública o la negociación de un contrato con una entidad pública, el inversor deberá tener cuidado con adquirir los servicios de "agentes" locales que afirman tener conexiones con altos funcionarios públicos o que ofrecen como parte

\footnotetext{
4 La sub-comisión de derechos humanos de las Naciones Unidas publicó en 2003 proyecto de código de conducta sobre responsabilidad social corporativa. addendum a la Declaración Universal de Derechos Humanos llamada "Human Rights Principles and Responsibilities for Transnational Corporations and Other Business Enterprises". La OECD ha tomado un papel importante en el debate sobre responsabilidad social corporativa. Véase por ejemplo OECD. Guidelines for Multinational Enterprises, Decision and Declaration on International Investment and Multinational Enterprises,2000.

5 Véase The Guidelines for Multinational Corporations, OECD Convention on Combating Bribery of Foreign Public Officials in International Business Transactions, 1997.
} 
de sus servicios la obtención de permisos y licencias. El inversor debe entender la diferencia entre pagar a alguien por asesoría en cómo hacer negocios en un determinado país y quien dice tener acceso a las cúpulas del gobierno y otra es pagar u otorgar favores a funcionarios públicos. El primero es legítimo y el último es soborno.

(iv) Proceder con cautela cuando se escoja un socio local. En muchas ocasiones, el Estado impondrá como condición que se establezca una empresa local y que haya participación local en el proyecto de inversión. En otras el inversor preferirá asociarse con un inversor local por consideraciones empresariales. El inversor buscará un socio con buena reputación en el país, conexiones y experiencia en el mercado local. Es común que algunos de los problemas en la operación de la inversión tengan que ver con problemas entre los socios accionistas. Por ello el inversor deberá revisar cuidadosamente las condiciones sobre propiedad y control accionaria en la ley corporativa local. El inversor podrá incluir cláusulas de protección en el contrato.

(v) Si se suscribe un contrato público revisar cuidadosamente la capacidad que cuenta la autoridad pública para la suscripción de dicho contrato. Es importante que el inversor verifique la capacidad de la autoridad para firmar contratos y las restricciones en la legislación que pudieren existir sobre la capacidad de dicha autoridad para someterse, en su caso, al arbitraje internacional.

En la etapa post-inversión, el inversor deberá:

(i) Mantener los estándares de operación que le son aplicables en su país de origen. Un inversor no debe relajar sus estándares de calidad en un país en desarrollo sólo porque en el país en el que invierte la regulación y aplicación de la ley es deficiente. Esto incluye la obligación de no sobornar a funcionarios públicos;

(ii) Cumplir con la ley local. El inversor debe asumir un estricto cumplimiento de la ley en materia de medio ambiente, salud, seguridad y demás normas y estándares de lo contrario será sujeto no sólo de sanciones administrativas (dependiendo de la seriedad de la infracción) sino también correrá el riesgo que dichas irregularidades sean utilizadas -junto con otras causas- para que la autoridad dé por terminada la operación.

(iii) Asumir dentro de sus políticas internas y en los contratos públicos conceptos de responsabilidad corporativa. Los inversores deben apoyar los objetivos de desarrollo en la comunidad en la que operan. Los gobiernos deberán asegurarse de incorporar dichos conceptos en los instrumentos que regulen la operación.

(iv) Asumir un compromiso de diálogo y no exacerbar un conflicto. 
En la etapa de conflicto con la autoridad: Establecer un esquema de manejo de disputas. Al momento de ocurrir un acto que el inversor considere vulnera sus derechos como inversor extranjero, el inversor deberá entablar comunicación y promover el diálogo con la autoridad responsable del acto y con la agencia del gobierno responsable de la prevención o promoción de las inversiones extranjeras. Es aconsejable que el inversor cuente con asesoría legal especializada en el manejo de disputas de inversión.

El inversor tiene un rol muy importante en la prevención de disputas de inversión. Especialmente las empresas multinacionales deben revisar sus políticas internas y asegurar que los estándares de conducta y valores que les son aplicables en sus países de origen los apliquen en sus operaciones en países en desarrollo. Deben asimismo realizar esfuerzos para asumir compromisos sociales y de diálogo con la comunidad en la que operan, especialmente cuando sus operaciones afectan sectores vulnerables de la sociedad.

El compromiso no es sólo del inversor. El gobierno y sociedad civil deberán asumir su rol en el cumplimiento de los objetivos de desarrollo social, fomentar el diálogo y evitar exacerbar un conflicto relativo a la operación de una inversión.

\section{Participación pública}

Para entender el grado de participación pública en el actuar del gobierno es imprescindible analizar la definición de gobernanza. El diccionario de la Real Academia Española (22a edición) define gobernanza como "el modo o arte de gobernar que se propone como objetivo el logro de un desarrollo económico, social e institucional duradero, promoviendo un sano equilibrio entre el estado, la sociedad civil y el mercado de economía". De esto se puede inferir que el desarrollo de una sociedad sólo se puede lograr mediante la acción conjunta entre la autoridad, la producción y los intereses de la sociedad civil. Esto es evidente e imperante en ciertos sectores como el medio ambiente y explotación de recursos naturales. Hoy en día es imposible imaginar que una autoridad responsable de la emisión de las licencias en la extracción de recursos naturales no mantenga un diálogo constante con la comunidad y la municipalidad en la que un determinado proyecto de inversión se lleve a cabo.

Un principio básico para que existan canales serios de participación pública en los proyectos de inversión con alto impacto en la comunidad es el de la transparencia. Para ello el ministerio o entidad pública deberá publicar en medios abiertos los proyectos de inversión que se tienen planeados y organizará seminarios y talleres dirigidos a la comunidad o la municipalidad no solamente con el objetivo de diseminar 
información respecto a los beneficios y alcances de un determinado proyecto de inversión sino también para educar a la comunidad sobre las obligaciones internacionales del Estado en materia de la protección de las inversiones extranjeras al amparo de los TBI.

Entre mayor participación pública mayor transparencia en el actuar de la autoridad y mejor informada será la decisión de la autoridad. A través de un mecanismo de prevención, no sólo se evitará o minimizará el riesgo de arbitrajes de inversión, sino fomentará la colaboración y cooperación entre las autoridades teniendo como resultado instituciones públicas más transparentes e incluyentes. Ello llevará como resultado un sistema de gobernanza que facilite la corrección institucional, el diálogo y entendimiento de todos los sectores dando como resultado el fin último de una política pública de atracción a la inversión: propiciar un clima favorable que fomente el desarrollo de los países de Latinoamérica.

\section{CONCLUSIÓN}

El presente artículo propone un esquema de prevención de disputas en proyectos de inversión con alto impacto social que crea y fortalece los canales de comunicación y cooperación entre las entidades de gobierno, la comunidad y las empresas privadas. Al mantener un esquema de cooperación interinstitucional en donde cada institución formula sus observaciones tomando en cuenta la opinión de la comunidad lleva como resultado un mejoramiento en el ejercicio de toma de decisiones de las autoridades. Bajo un esquema de mayor transparencia, comunicación y cooperación gubernamental, el sistema de gobernabilidad de los países de Latinoamérica mejorará y por ende se generará un clima favorable para la inversión.

\section{REFERENCIAS BIBLIOGRÁFICAS}

OECD. "The Guidelines for Multinational Corporations". In: Convention on Combating Bribery of Foreign Public Officials in International Business Transactions (Nov 1997). s.l: s.n., 1997.

OECD. Guidelines for Multinational Enterprises, Decision and Declaration on International Investment and Multinational Enterprises. s.l: s.n, 2000. REAL ACADMIA ESPAÑOLA. Diccionario de la lengua española. 22a ed. Madrid: RAE, 2012. 